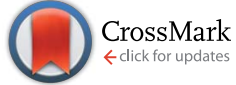

Cite this: J. Mater. Chem. A, 2017, 5, 2689

Received 14th October 2016 Accepted 16th December 2016

DOI: $10.1039 / c 6 t a 08950 j$

www.rsc.org/MaterialsA

\section{Origin of fullerene-induced vitrification of fullerene:donor polymer photovoltaic blends and its impact on solar cell performance $\uparrow$}

Paul Westacott, ${ }^{a}$ Neil D. Treat, ${ }^{a}$ Jaime Martin, ${ }^{a}$ James H. Bannock, ${ }^{b}$ John C. de Mello, Michael Chabinyc, ${ }^{c}$ Alexander B. Sieval, ${ }^{d}$ Jasper J. Michels ${ }^{* e}$ and Natalie Stingelin*af

Organic solar cell blends comprised of an electron donating polymer and electron accepting fullerene typically form upon solution casting a thin-film structure made up of a complex mixture of phases. These phases can vary greatly in: composition, order and thermodynamic stability; and they are dramatically influenced by the processing history. Understanding the processes that govern the formation of these phases and their subsequent effect on the efficiency of photo-generating and extracting charge carriers is of utmost importance to enable rational design and processing of these blends. Here we show that the vitrifying effect of three fullerene derivatives ([60]PCBM, bis[60]PCBM, and [60]ICBA) on the prototypical donor polymer ( $r$-P3HT) can dominate microstructure formation of fullerene/donor polymer blends cast from solution. Using a dynamic crystallization model based on an amalgamation of Flory-Huggins and Lauritzen-Hoffman theory coupled to solvent evaporation we demonstrate that this vitrification, which can result in a large fraction of highly intermixed amorphous solid solution of the fullerene and the polymer, is due to kinetic and thermodynamic reasons. The former is partly determined by the glass transition temperature of the individual components while donor polymer:fullerene miscibility, strongly influenced by the chemical nature of the donor and the fullerene and leading to thermodynamic mixing, dictates the second phenomena. We show that our approximate dynamic crystallization model assists understanding the different solid-state structure formation of rr-P3HT:fullerene blends. Due to the generality of the assumptions used, our model should be widely applicable and assist to capture the influence of the different vitrification mechanisms also of other photovoltaic blends, including the high-efficiency systems based on the strongly aggregating PCE11 (PffBT4T-2OD), which also feature clear signs of vitirfication upon blending with, e.g., [60]PCBM. Hence, our model will provide essential materials design criteria and enable identification of suitable processing guidelines for existing and new high-performing blends from the outset.
Organic photovoltaics (OPVs) have seen a rapid increase in performance over recent years, with certain polymer:fullerene blends now reaching efficiencies of more than $10 \%$. This improvement has mainly been due to intense materials

\footnotetext{
${ }^{a}$ Department of Materials and Centre for Plastic Electronics, Imperial College London, London, SW7 2AZ, UK

${ }^{b}$ Department of Chemistry and Centre for Plastic Electronics, Imperial College London, London, SW7 2AZ, UK

'Materials Research Laboratory, University of California Santa Barbara, CA 93106, USA

${ }^{d}$ Solenne BV, Zernikepark 6, 9747AN Groningen, The Netherlands

${ }^{e}$ Max Planck Institute for Polymer Research, Ackermannweg 10, 55128 Mainz, Germany.E-mail: michels@mpip-mainz.mpg.de

${ }^{f}$ School of Materials Science and Engineering, School of Chemical \& Biomolecular Engineering, Georgia Institute of Technology, 311 Ferst Drive, Atlanta, Georgia 30332, USA. E-mail: natalie.stingelin@mse.gatech.edu

$\dagger$ Electronic supplementary information (ESI) available. See DOI: $10.1039 /$ c6ta08950j
}

development efforts. Despite these activities, however, key understanding of various relevant aspects that dictate the structural and optoelectronics landscape of many OPV materials, is still lacking. For instance, it is still unclear why specific acceptors work well only in combination with certain donors. ${ }^{1}$ Moreover, thorough knowledge has not been established why small variations in the chemical structure of the active materials in certain cases can lead to significant differences in device performance while in other scenarios essentially identical device characteristics are obtained. ${ }^{2}$

One reason for these differences that are observed in devices even for structurally very similar materials is that the manipulation of the chemical structure often results in alteration of the energy levels, ${ }^{\mathbf{1}, 3}$ which, in the case of the LUMO of the acceptor, should either promote charge dissociation or increase the open circuit voltage $\left(V_{\text {oc }}\right)$ with a given donor. Chemical changes lead, however, also to a different miscibility of the two components, 
which can affect OPV device characteristics considerably, ${ }^{4}$ as well as a different thermal behaviour. There is, hence, a strong drive to improve our understanding of the interplay of: solubility in a given solvent, mutual miscibility of the two components and their aggregation/crystallisation behaviour, on the final photo-physical properties of such donor:acceptor blends. More specifically, in-depth insights are required about the solubility of the donor and acceptor in a given solvent and how this influences the solidification of each material during solution casting and, indeed, their solidification sequence. This in turn strongly determines to what extent the materials can molecularly order and whether kinetic and/or thermodynamic vitrification dominates solidification, which can result in the formation of a highly intermixed solid solution that is in most cases amorphous. Conversely, if the materials crystallise - at least partly - during casting, knowledge has to be obtained whether coarse phase separation occurs, resulting often in the formation of large, relatively phase-pure domains.

In order to elucidate how these phenomena and resulting phase morphologies affect the processes relevant in photovoltaic devices, we selected donor:acceptor systems utilising three different $\mathrm{C}_{60}$ fullerene derivatives as the acceptor, namely, [6,6]-phenyl- $\mathrm{C}_{61}$-butyric acid methyl ester ([60]PCBM), bis(1-[3-(methoxycarbonyl)propyl]-1-phenyl)-[6.6] $\mathrm{C}_{62}$ (bis[60] PCBM), and $1^{\prime}, 1^{\prime \prime}, 4^{\prime}, 4^{\prime \prime}$-tetrahydro-di[1,4]methanonaphthaleno $[5,6]$ fullerene- $\mathrm{C}_{60}([60] \mathrm{ICBA})$ (chemical structures are shown in Fig. 1A). These show significant promise in terms of both device performance $^{5}$ and for scale-up of production. Also, since these fullerenes have different chemical structures, they are expected to exhibit different miscibility with the same donor polymer. Furthermore, as these three fullerene derivatives are known to have relatively high glass transition temperatures, $T_{\mathrm{g}},{ }^{6}$ we might also encounter differences in the kinetically driven vitrification of the solution cast blend films - all factors that can strongly affect the solid-state microstructure formation of such blends.

As the donor, we chose a polymer with well understood and measureable crystallisation behaviour, i.e. regioregular poly(3hexylthiophene-2,5-diyl) (rr-P3HT). This allows for a clear comparison of the effect of the respective fullerenes on the formation of molecularly ordered domains within the polymer and the resulting fractions of mixed and pure phases that will be present in the final solid-state structure. Furthermore, rr-P3HT, unlike many polymers shows distinct phase transitions observable during thermal analysis, thereby providing an additional tool to probe donor:acceptor compatibility, as well as structural characteristics of the resulting blends. A relatively high molecular weight material was utilised $\left(M_{\mathrm{w}} \approx 135 \mathrm{~kg} \mathrm{~mol}^{-1}, \mathrm{PDI} \approx 1.9\right)$ to further drive vitrification. Gained knowledge should however be applicable to other donor:acceptor systems, including higher performing systems, as many will show either kinetically or thermodynamically driven vitrification - or both.

We first set out to gain an initial understanding of the fullerene's influence on structure formation in the corresponding blends, by studying the thermal behaviour of the selected rr-P3HT:fullerene systems using differential scanning calorimetry (DSC). To this end, we prepared solution-cast films of rrP3HT:fullerene mixtures with varying donor-acceptor ratio from a solvent mixture of chlorobenzene (CB) containing $1.5 \mathrm{vol} \%$ of 1-chloronaphthalene $(\mathrm{CN})$. Crystallization was allowed to proceed at room temperature under evaporative conditions. Prolonged drying in vacuum (without heating, see Experimental section) guaranteed removal of (co-)solvent residues. Subsequently, we determined the polymer's melting temperature $\left(T_{\mathrm{m}}\right)$ as a function of fullerene content using the end of melting temperatures.

In Fig. 1B, the thermograms (first heating) for various rr-P3HT:fullerene blends are shown. Independent of what fullerene we used, a clear depression of the non-equilibrium melting temperature of rr-P3HT with increasing acceptor fraction is observed (Fig. 1B), with the depression increasing in the order: bis[60]PCBM $<$ [60]PCBM $<$ [60]ICBA. In Fig. 1C we plot the end-of-melting temperature as a function of the (approximate) volume fraction of fullerene. The conversion from weight to volume fraction was accomplished using the straightforward relation for a binary system: $\varphi_{1}=\frac{w_{1}}{\rho_{1}} /\left[w_{1}+\left(1-w_{1}\right) / \rho_{2}\right]$, with $\varphi$ and $w$ respectively representing volume and weight fraction. The mass densities of polymer ${ }^{7}$ and fullerene ${ }^{8}$ were taken to be $\sim 1$ and $\sim 1.5 \mathrm{~g} \mathrm{~cm}^{-3}$, respectively. ${ }^{9}$

A depression in the non-equilibrium melting temperature has two major contributions: (i) thermodynamic: ${ }^{10}$ lowering of the supercooling due to depression of the equilibrium melting temperature upon blending, and (ii) kinetic: the size of the crystallites remaining limited due to kinetic vitrification of the amorphous fractions. In donor:acceptor binaries, the latter means that upon blending the crystallization process of at least one component may be prohibited (or be at least strongly hindered), leading to the formation of a highly intermixed, quenched 'solid solution', wherein a substantial fraction of the polymer is frozen into a finely intermixed state with the fullerene, as has been shown for rr-P3HT:[60]PCBM binaries. ${ }^{\mathbf{1 1}}$

In order to describe the depression in the non-equilibrium melting point, we constructed an approximate model for rr-P3HT crystallization including thermodynamic as well as kinetic aspects. The model subdivides the crystallization process in two stages: (i) an isothermal stage representing drying of the solution-cast film, and (ii) a subsequent non-isothermal stage representing the first heating run in the DSC. The primary aim of this exercise is not to provide a detailed mechanistic treatment of crystallization and melting during the various processing and analysis steps, but rather to allow for internal comparison between the three blend systems of the relative importance of thermodynamic and kinetic melting point depression of rr-P3HT and to provide a morphological basis for explaining their photophysical performance.

We start with the relation between the size of a rectangular crystallite of a flexible polymer and its melting point, as given by the Gibbs-Thompson equation: ${ }^{\mathbf{1 2}}$

$$
T_{\mathrm{m}}=T_{\mathrm{m}}^{\mathrm{o}}\left[1-\frac{2}{\Delta h}\left(\frac{\sigma_{\mathrm{e}}}{L}+\frac{\sigma_{x}}{y}+\frac{\sigma_{y}}{x}\right)\right],
$$

where $T_{\mathrm{m}}$ and $T_{\mathrm{m}}^{\mathrm{o}}$ are, respectively, the observed and equilibrium melting temperature, and $\Delta h$ is the molecular enthalpy of crystallisation per unit volume. $L, x$, and $y$ are the (lamellar) thickness (i.e. the length of an extended chain segment in 
A

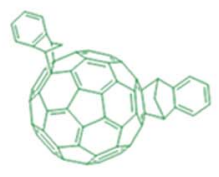

B

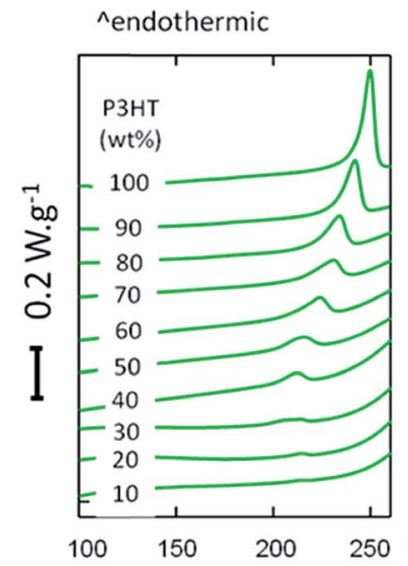

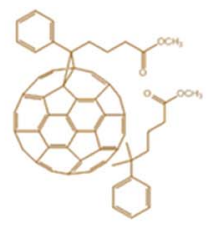

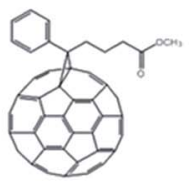

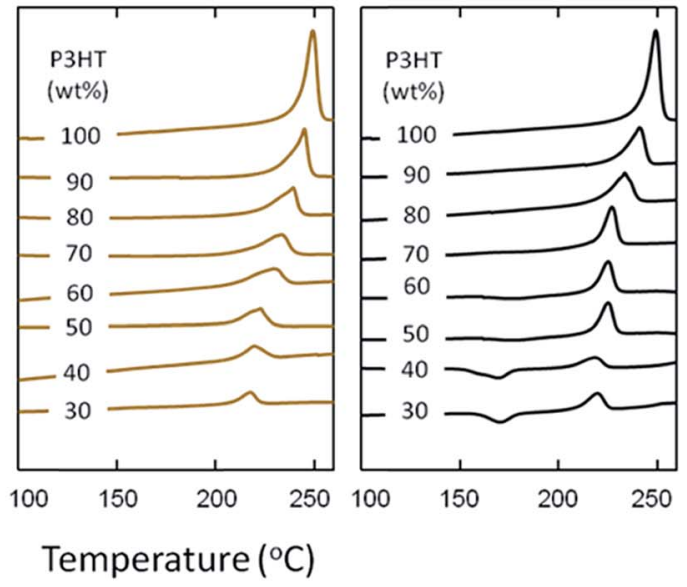

C

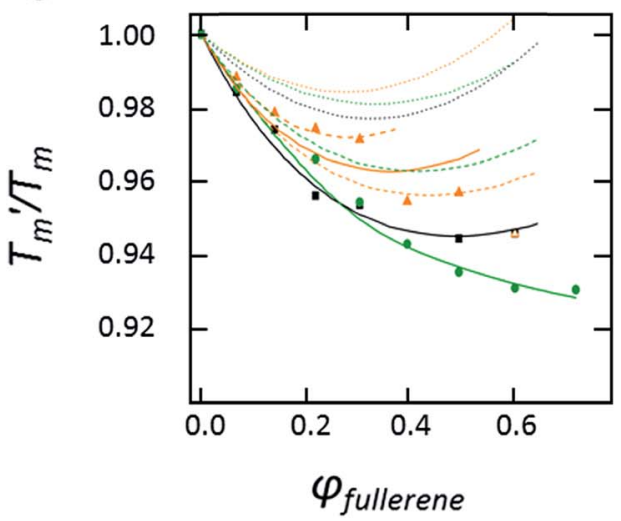

D

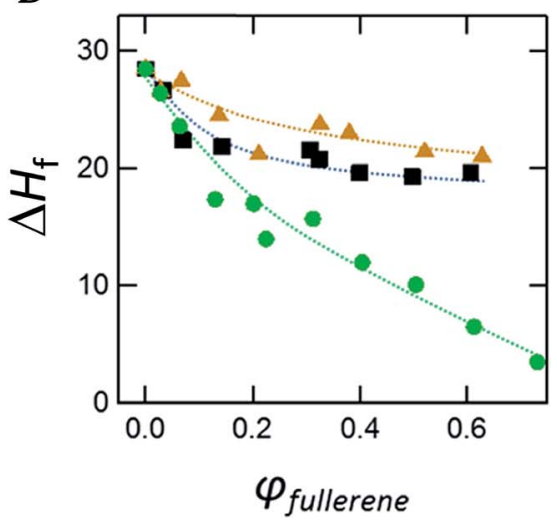

Fig. 1 Thermal behaviour of three rr-P3HT:fullerene blends, showing clear differences between the binaries systems. (A) Chemical structures of the fullerenes investigated here: [60]PCBM (left, squares), bis[60]PCBM (middle, triangles) and [60]ICBA (right, circles). The same symbols are used for each fullerene throughout this manuscript. (B) Differential scanning calorimetry first heating thermograms (heating rate $=10^{\circ} \mathrm{C}$ min ${ }^{-1}$ ) of rr-P3HT:fullerene binaries cast from solution and of a rr-P3HT content as indicated: [60]ICBA (left), bis[60]PCBM (middle) and [60]PCBM (right). (C) Observed rr-P3HT melting temperature plotted as a function of fullerene volume fraction: [60]PCBM (black), bis-[60]PCBM (orange), [60]ICBA (green); points indicate measured values obtained from a first DSC heating run; lines are calculations using the model described below: bold/solid and bold/dashed: best fit, thin/dashed: assuming the interaction of bis-[60]PCBM and [60]ICBA with rr-P3HT to be similar to [60] PCBM, thin/dotted: similar interaction and hypothetically assuming $T_{g}$ (fullerene) $=T_{g}(\mathrm{P} 3 H T)$. (D) Enthalpy of fusion of rr-P3HT $\left(\Delta H_{f}^{P 3 H T}\right)$ as measured for the different binaries with [60]ICBA (green), bis[60]PCBM (yellow) and [60]PCBM (black). The $\Delta H_{f}^{\text {PHTT }}$ was extracted from the integral of the melting endotherm and normalised for the mass of polymer in the sample, thus, enabling comparison of the degree of crystallinity of the polymer in the different blends.

a crystalline moiety) and the lateral dimensions of the crystallite; $\sigma_{\mathrm{e}}, \sigma_{x}$, and $\sigma_{y}$ are the surface energies of the terminal ("end") and lateral faces. Here, the former are referred to as the ones that accommodate loops and folds in the polymer chains exiting and re-entering the crystalline lamella. If the polymer chain is axisymmetric, as is the case for various "classical" flexible semi-crystalline polymers such as polyethylene and poly(vinylidene fluoride) (PVDF), we write: $\sigma_{x}=\sigma_{y}$. In this work the equilibrium melting point may either refer to the neat polymer $\left(T_{\mathrm{m}}^{\mathrm{o}}\right)$, or to a mixed state $\left(T_{\mathrm{m}}^{\mathrm{o} \prime}\right)$ in which the crystallisable polymer has been blended with one or more amorphous diluents, such as solvent or fullerene.

To calculate the relation between $T_{\mathrm{m}}^{\mathrm{o}}$ and $T_{\mathrm{m}}^{\mathrm{ol}}$ we invoke an extended version of the generalized Flory-Scott equation: $:^{10,13}$

$$
\begin{aligned}
\frac{1}{T_{\mathrm{m}}^{\mathrm{o}}}-\frac{1}{T_{\mathrm{m}}^{\mathrm{o}}}= & -\frac{R V_{\mathrm{u}}}{\Delta H_{\mathrm{c}} V_{\mathrm{seg}}}\left[1-\varphi_{j}+\frac{\ln \varphi_{j}}{N_{j}}-\sum_{i \neq j}^{n} \frac{\varphi_{i}}{N_{i}}\right. \\
& \left.+\left(1-\varphi_{j}\right) \sum_{i \neq j}^{n} \chi_{i j} \varphi_{i}-\sum_{i \neq k \neq j}^{n} \chi_{i k} \varphi_{i} \varphi_{k}\right] .
\end{aligned}
$$


Here, $\varphi_{j}$ indicates the volume fraction of crystallisable polymer; $\varphi_{i}$ and $\varphi_{k}$ represent volume fractions of the amorphous constituents in the $n$-component blend; ${ }^{14} \mathrm{~N}$ represents effective molecular size, $\chi$ is the (temperature-dependent) Flory interaction parameter, $\Delta H_{\mathrm{c}}$ is the molar enthalpy of crystallisation, $V_{\mathrm{u}}$ the molar volume of a crystallisable repeat unit, and $V_{\text {seg }}$ a reference volume, for instance the molar volume of the smallest component in the diluent mixture (in our case chlorobenzene), and $R$ the gas constant.

In order to model the non-equilibrium melting point depression, we note that eqn (1) and (2) do not suffice as (i) the polymer typically exhibits a melting range partly due to the fact that $\mathrm{L}$ is not spatially constant, and (ii) kinetic factors that affect $L$ during solvent evaporation are not taken into account. Instead, we seek an effective distribution function for the lamellar thickness, with its first moment indicating the most abundant value. Expressions are required that describe the change of this distribution function with time, whereby three stages are being considered: (i) primary nucleation, (ii) linear crystal growth, and (iii) melting. For simplicity, non-isothermal secondary thickening is not considered. Besides a description of the crystallization kinetics, a treatment is required that allows for changes in composition e.g. due to solvent evaporation.

Primary nuclei are assumed to already form at low concentration, whereby we consider recent cryo-TEM work ${ }^{\mathbf{1 5}}$ demonstrating significant rr-P3HT crystallization at $\sim 1 \mathrm{wt} \%$ concentration in support of this assumption. The free energy of a nucleus with dimensions $x \times y \times L$ is written as:

$$
\Delta G=-x y L \Delta \mu+2\left(x y \sigma_{\mathrm{e}}+x L \sigma_{x}+y L \sigma_{y}\right)
$$

with $\Delta \mu$ the free energy of crystallization per unit volume approximated by: $\Delta \mu \approx \Delta h\left(T_{\mathrm{m}}^{\mathrm{o} \prime}-T\right) / T_{\mathrm{m}}^{\mathrm{o}}$. The free energy of formation $\Delta G_{\text {nuc }}^{*}$ of such a nucleus (via homogeneous nucleation) is for given $x$ and $y$ straightforwardly obtained as a function of $L$ by solving $\partial_{x} \Delta G=0$ and $\partial_{y} \Delta G=0$ for $x$ and $y$ and substituting back into the expression for the free energy. The result is:

$$
\Delta G_{\mathrm{nuc}}^{*}(L)=\frac{4 L^{2} \sigma_{x} \sigma_{y}}{L \Delta \mu-2 \sigma_{\mathrm{e}}} .
$$

Eqn (4) allows for the definition of an approximate distribution function for the lamellar thickness:

$$
f_{\text {nuc }}(L) \propto \exp \left[-\frac{\Delta G_{\text {nuc }}^{*}(L)}{k T}\right] .
$$

Growth of the nuclei beyond their critical size yields stable crystallites that eventually enter a regime of linear growth.

A well-known model describing linear growth of crystals of flexible polymers is the Lauritzen-Hoffman ( $\mathrm{LH}$ ) model. ${ }^{16}$ This model is valid if crystallization is dominated by tight chain folding. Linear crystal growth is assumed to take place via surface nucleation of subsequent layers of extended chain segments ("stems") onto the growth face(s) of the crystallites. The model considers an ensemble of crystals, each growing with a constant but individually different lamellar thickness. The theory provides for the definition of a distribution function for the growth rate based on lamellar thickness, of which the first moment corresponds to the most abundant value for $L$.

Despite certain criticism ${ }^{16 c}$ concerning its somewhat artificial representation of the ensemble, the LH model has shown to accurately reproduce experimental observations and owing to its conceptual and computational tractability continues to provide a popular analytical framework for interpreting experimental crystal growth data, as has for instance been demonstrated for poly( $\varepsilon$-caprolactone $)^{17}$ and PVDF. ${ }^{18}$ rr-P3HT also exhibits sufficient chain flexibility ${ }^{\mathbf{1 9 2 0}}$ to allow for considerable chain folding (as long as $M_{\mathrm{n}}>10 \mathrm{kDa}$ ), especially if crystallization is given sufficient time. ${ }^{21}$ Since in our experiments, crystallisation occurs from relatively slowly evaporating solution-cast films, we suggest chain folding to be extensive and, hence, consider the LH model applicable.

Rephrasing the above more precisely, LH theory defines a distribution function for the steady state flux $S(L)\left(\right.$ in s $\left.^{-1}\right)$ of crystallising extended chain segments with length $L$ towards the crystalline substrate:

$$
S(L)=\frac{\varphi_{j} N_{0} A_{0}(A-B)}{A-B+B_{1}},
$$

where $A_{0}$ and $A$ are attachment rates of first and subsequent stems, and $B_{1}$ and $B$ are detachment rates of first and subsequent stems. $N_{0}$ represents the number of vacant positions at the growth front, ${ }^{22}$ obtained by dividing the growth front surface area by the area occupied by a single repeat unit. The flux equation is multiplied by the polymer volume fraction $\varphi_{j}$ if crystallization takes place from solution (instead of the melt).

In writing expressions for the attachment and detachment rates, it is instructive to realize that the rr-P3HT chain is not axisymmetric which in principle gives rise to two distinct growth faces for the $\pi-\pi$ and alkyl-stacking directions. Following the LH-protocol, we write the first stem attachment rate for the two faces as:

$$
\begin{aligned}
& A_{0}^{\pi-\pi}=\beta \exp \left(-\frac{2 b_{0} L \sigma_{\mathrm{alkyl}}-\psi a_{0} b_{0} L \Delta \mu}{k T}\right), \\
& A_{0}^{\mathrm{alkyl}}=\beta \exp \left(-\frac{2 b_{0} L \sigma_{\pi-\pi}-\psi a_{0} b_{0} L \Delta \mu}{k T}\right) .
\end{aligned}
$$

The attachment rates of subsequent stems as well as the detachment rates $(B)$ do not depend on the surface energy of the growth faces:

$$
\begin{gathered}
A^{\pi-\pi}=A^{\mathrm{alkyl}}=\beta \exp \left(-\frac{2 a_{0} b_{0} \sigma_{\mathrm{e}}-\psi a_{0} b_{0} L \Delta \mu}{k T}\right), \\
B_{(1)}^{\pi-\pi}=B_{(1)}^{\mathrm{alkyl}}=\beta \exp \left[-\frac{(1-\psi) a_{0} b_{0} L \Delta \mu}{k T}\right] .
\end{gathered}
$$

In eqn (7)-(10) $\psi$ represents an apportioning coefficient ${ }^{23}$ tuning the height of the activation barrier and $a_{0}$ and $b_{0}$ the width of the rr-P3HT chain in the alkyl and $\pi-\pi$ stacking 
direction. $\beta$ represents a kinetic retardation factor, which at temperatures near glass transition (i.e. $T-T_{\mathrm{g}}<100 \mathrm{~K}$ ) and may be given by a Vogel-Fulcher-Tammann-type expression: ${ }^{24}$

$$
\beta=\frac{\kappa}{n} \frac{k T}{h} \exp \left\{-\frac{U^{*}}{R\left[T-\left(T_{\mathrm{g}}-T_{0}\right)\right]}\right\},
$$

or in the Arrhenius regime $\left(T-T_{\mathrm{g}}>100 \mathrm{~K}\right) \mathrm{by:}:^{24}$

$$
\beta=\frac{\kappa}{n} \frac{k T}{h} \exp \left\{-\frac{U^{*}}{R T}\right\}
$$

with $k T / h$ a jump frequency, $U^{*}$ the activation energy for chain segmental motion, and $T_{\mathrm{g}}-T_{0}$ the temperature at which segmental motion is considered fully imparted; $n$ is the number of chemical repeat units in a polymer chain $\left(\sim 10^{3}\right.$ in the present case) and $\kappa$ a dimensionless factor of order unity resulting from friction experienced by the polymer chain once it is being "reeled" out of the amorphous phase due to the force exerted by crystallization. $^{22 a, 24 a}$ For the detailed definition of the stem attachment and detachment rates we refer to literature. ${ }^{\mathbf{1 6 b , 2 4 a , 2 5}}$ Moreover, it has been shown experimentally that under the present casting conditions higher molecular weight rr-P3HT (molecular weight above the entanglement limit) forms chainfolded nanofibrillar crystals with one-dimensional growth in the $\pi-\pi$ stacking direction..$^{20}$

Instead of taking the usual approach of calculating a total surface nucleation rate by integrating eqn (6) we consider the actual ensemble of crystals evolving in time under isothermal or non-isothermal conditions, either via growth or melting. Loosely phrased, this approach superimposes the growth distribution function, which changes as function of time and temperature, onto the initial Boltzmann distribution of the primary nuclei. ${ }^{26}$ We define the growth rate in the $\pi$-stacking direction $(x)$ as: ${ }^{27}$

$$
\frac{\mathrm{d} x}{\mathrm{~d} t}=a_{0} b_{0} S(L) / y,
$$

with $y$ being the width of the growth front situated parallel to the alkyl stacking dimension (making $x$ the $\pi$-stacking dimension indicating the growth direction). The present model is insensitive to the exact initial choice of $x$ and $y$, as long as they are sufficiently large to avoid re-dissolution of the nuclei. We choose $x=y=15 \mathrm{~nm}$ (ref. 20) and keep $y$ constant for $t>t_{0}$ to allow for the growth of nanofibrillar crystals. In the present coarse representation an intermediate transient regime during which the eventual width of the growth front is established is not considered. A simple but intuitive approach to melting is provided for realizing that $A<B$ once $T>T_{\mathrm{m}}$. In LH-terminology this results in a negative "substrate completion rate" $g=a_{0}(A-B)$, effectively inverting the "growth" direction of crystals that obey the condition: $L<2 \sigma_{\mathrm{e}} / \Delta \mu$.

Finally, we implement treatments for solvent evaporation and amorphous vitrification. Relative evaporation rates of $\mathrm{CB}$ and $\mathrm{CN}$ are calculated assuming the vapour phase just above the liquid film to be in equilibrium with local partial pressure scaling linearly with activity. Activity coefficients are calculated using the Flory-Huggins model ${ }^{10}$ assuming both $\mathrm{CB}$ and $\mathrm{CN}$ to be good solvents for both rr-P3HT and the fullerenes (i.e. $\chi<0.5$ ). As now the composition as a function of (relative) time is known, we can estimate the instantaneous glass transition temperature of the amorphous mixture using the Fox equation..$^{28}$ For this we require the glass transition temperatures of the neat components (see Table 1). The $T_{\mathrm{g}}$ 's of $\mathrm{CB}$ and $\mathrm{CN}$ are unknowns. They are, hence, used as free fitting parameters but constrained to be lower than the melting temperatures ( 228 and $259 \mathrm{~K}$, respectively).

The model is implemented by updating temperature and system-related parameters in a numerical time integration procedure involving two stages: (i) an isothermal stage ( $T=$ $293 \mathrm{~K}$ ) characterized by slow solvent evaporation (on an arbitrary time scale), followed by (ii) a heating stage (293-573 K), representing the DSC run (at a rate of $\sim 0.17 \mathrm{~K} \mathrm{~s}^{-1}$ ). The size evolution of crystals with lamellar thicknesses in the range $0.1<$ $L<30 \mathrm{~nm}$ is tracked in time by storing both the individual and total volume of crystallites at regular time intervals. At the end of the simulation run the time differential in the total crystal volume $(\delta V)$ is calculated at each recorded time and temperature. The melting point is taken to be the first moment of the resulting distribution function: ${ }^{29}\left\langle T_{\mathrm{m}}\right\rangle=\int T \delta V \mathrm{~d} T / \int \delta V \mathrm{~d} T$.

In view of the approximate nature of the model, we scale the melting point obtained at non-zero fullerene loading by that of neat rr-P3HT instead of considering absolute values. The

\begin{tabular}{|c|c|c|c|c|c|c|}
\hline Parameter & P3HT & PCBM & Bis-BCBM & ICBA & $\mathrm{CB}$ & $\mathrm{CN}$ \\
\hline$M\left(\mathrm{~g} \mathrm{~mol}^{-1}\right)$ & 135.000 & 910.88 & 1101.1 & 953.4 & 112.56 & 162.62 \\
\hline$\rho\left(\mathrm{g} \mathrm{cm}^{-3}\right)$ & 1 & 1.5 & 1.5 & 1.5 & 1.11 & 1.19 \\
\hline$N$ & 1331 & 6.0 & 7.2 & 6.3 & 1 & 1.3 \\
\hline$a_{0}(\AA)$ & $16.6^{a}$ & - & - & - & - & - \\
\hline$b_{0}(\AA)$ & $7.75^{a}$ & - & - & - & - & - \\
\hline$l_{\mathrm{u}}(\AA)$ & 1 & - & - & - & - & - \\
\hline$V_{\mathrm{u}}\left(\mathrm{cm}^{3} \mathrm{~mol}^{-1}\right)$ & $151^{b}$ & - & - & - & - & - \\
\hline$\sigma_{\mathrm{e}}\left(\mathrm{J} \mathrm{m}^{-2}\right)$ & $0.1^{c}$ & & & & & \\
\hline$\sigma_{\text {alkyl }}\left(\mathrm{J} \mathrm{m}^{-2}\right)$ & $0.01^{c}$ & & & & & \\
\hline$\sigma_{\pi-\pi}\left(\mathrm{J} \mathrm{m}^{-2}\right)$ & 0.02 & & & & & \\
\hline$T_{\mathrm{m}}^{\mathrm{o}}(\mathrm{K})$ & $571^{d}$ & - & - & - & - & - \\
\hline$T_{\mathrm{m}, \text { nuc }}(\mathrm{K})$ & 293 & - & - & - & - & - \\
\hline$T_{0}$ & $20-25$ & - & - & - & - & - \\
\hline$T_{\mathrm{g}}(\mathrm{K})$ & $283^{e}$ & $393^{e}$ & $418^{f}$ & $441^{f}$ & 200 & 170 \\
\hline $\begin{array}{l}\Delta H_{\mathrm{c}} \\
\left(\mathrm{kJ} \mathrm{mol}^{-1}\right)\end{array}$ & 12 & - & - & - & - & - \\
\hline $\begin{array}{l}U_{\mathrm{P} 3 \mathrm{HT}}^{*} \\
\left(\mathrm{~kJ} \mathrm{~mol}^{-1}\right)\end{array}$ & - & 5 & 5 & 5.5 & - & - \\
\hline$\psi_{0}$ & - & 0.37 & 0.37 & 0.35 & & \\
\hline$\psi_{1}$ & - & 0.34 & $0.1(0.2)$ & $\boldsymbol{O}$ & & \\
\hline$\chi_{\text {Р3HT- }}$ & - & $0.4^{g}$ & $0.5^{h}\left(0.6^{i}\right)$ & 0.1 & 0.4 & $\boldsymbol{O}$ \\
\hline$\chi_{\text {PCBM- }}$ & 0.4 & - & - & - & 0.1 & $\boldsymbol{O}$ \\
\hline$\chi_{\text {bisPCBM- }}$ & $0.5(0.6)$ & - & - & - & 0.1 & $\boldsymbol{O}$ \\
\hline$\chi_{\text {ICBA- }}$ & 0.1 & - & - & - & 0.1 & $\boldsymbol{O}$ \\
\hline$\chi_{\mathrm{CB}-}$ & 0.4 & 0.1 & 0.1 & 0.1 & - & $\boldsymbol{O}$ \\
\hline & 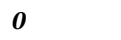 & $\boldsymbol{O}$ & $\boldsymbol{O}$ & $\boldsymbol{O}$ & $\boldsymbol{O}$ & - \\
\hline
\end{tabular}

Table 1 Fixed (normal type) and free floating (bold/italic) model parameters

${ }^{a}$ From ref. $30 .{ }^{b}$ From ref. $31{ }^{c}$ From ref. $21 b .{ }^{d}$ Equilibrium melting point of "Type I" rr-P3HT crystals, as reported in ref. $33 .{ }^{e}$ From ref. 32 and 33. ${ }^{f}$ From ref. $6 .{ }^{g} \chi_{\text {P3HT-РСBM }} \approx 0.3$ assuming $\rho_{\text {fullerene }}=$ $1.3 \mathrm{~g} \mathrm{~cm}^{-3}$ (see ref. 9). ${ }^{h} \chi_{\mathrm{P} 3 \mathrm{HT}-\text { bisPCBM }} \approx 0.4$ assuming $\rho_{\text {fullerene }}=$ $1.3 \mathrm{~g} \mathrm{~cm}^{-3} \cdot{ }^{i} \chi_{\text {P3HT-bisPCBM }} \approx 0.5$ assuming $\rho_{\text {fullerene }}=1.3 \mathrm{~g} \mathrm{~cm}^{-3}$. 
calculations were performed in a simultaneous fitting procedure of all melting point depression data (Fig. 1C). Model parameters were as much as possible retrieved from literature or fixed through measurement. Remaining parameters were treated freely floating, however constrained to remain within reasonable physical limits. The best fit result corresponds to the bold solid (black, green) and bold dashed (orange) lines in Fig. 1C with all associated parameter values are listed in Table 1. On the inclusion of the data points obtained from the heating runs we note that for the $90 \mathrm{wt} \%(\sim 86$ vol\%) ICBA:P3HT blend a clear "end-of-melting" temperature was indiscernible due to the very low signal-to-noise ratio. Furthermore, fitting of the P3HT:bis-PCBM data turned out somewhat ambiguous in comparison to P3HT:PCBM and P3HT:ICBA. At bis-PCBM loadings exceeding $40 \mathrm{wt} \%$ the recorded melting signal exhibits pronounced variation in shape; including the point corresponding to $\varphi_{\text {bisPCBM }}=61 \mathrm{vol} \%$ (open orange triangle) led to physically unrealistic results. The exact reason for the fluctuation is unclear. Only including data points corresponding to a low bis-PCBM loading, for which a minor contribution from the approach to glass transition is expected, resulted in the dashed orange line in Fig. 1C and corresponds to the values between brackets in Table 1 .

Before discussing the values listed in Table 1 a few remarks concerning the calculation are in place. First, the LH model assumes crystallization to take place in an infinite bath of the amorphous blend and therefore does not allow for the calculation of the ratio of crystalline to amorphous material in a particular sample. Second, it is assumed that the crystal surface energies do not vary with solvent or fullerene content, as might be expected considering ref. $21 b$. Nevertheless, the fit results appear relatively insensitive towards moderate variations in surface energy. Third, the activation barrier for an approach of chain segments to and from the crystalline face $\left(U_{\mathrm{P} 3 \mathrm{HT}}^{*}\right)$ is considered independent of polymer concentration, reasoning that the value does not vary greatly as crystallization predominantly takes place in a highly concentrated regime nearly devoid of CB.

The Flory-Huggins interaction parameters obtained from curve fitting (Table 1) suggest the (thermodynamic) miscibility between rr-P3HT and fullerene to increase in the order bis[60] PCBM $<[60] \mathrm{PCBM}<[60] \mathrm{ICBA}$, whereby we note that the reduced rr-P3HT melting point depression in case of bis[60] PCBM as compared to [60]PCBM and [60]ICBA is expected to be partly of entropic origin due to bis[60]PCBM's significantly larger molecular volume. The obtained rr-P3HT:fullerene Flory parameters are of comparable magnitude to values recently calculated for the same blend systems using the Hansen solubility parameter approach. ${ }^{6}$ Interestingly, though, the order of thermodynamic miscibility reported by these authors, bis[60]PCBM $<$ [60]ICBA $<$ [60]PCBM, is only partially consistent with our results, the exact reason being unclear at present.

We also note that the $\chi$-values obtained in this work (specifically $\chi_{\text {P3HT-PCBM}}$ ) are lower than the one found by Kozub et al. $\left(\chi_{\text {Р3нт-РСBM }}=0.86\right)$ also using thermal analysis and deducing the melting point depression of P3HT upon addition of [60]PCBM. ${ }^{31}$ There can be multiple reasons for this discrepancy. We used materials from cast films and deduced values from the 1st heating scan, while specific details are not given in ref. 31. In addition, small variations in regioregularity, dispersity, molecular weight, etc., will affect the miscibility of a given polymer with a second component and, hence, $\chi$. We are moreover somewhat hesitant making a thorough comparison because Kozub et al. did not specifically address the fact that evaluation of $\chi$ using eqn (2) requires the equilibrium melting temperatures (corresponding to $L \rightarrow \infty$ ). Equilibrium melting points are usually obtained using the Hoffman-Weeks extrapolation method, ${ }^{34}$ however this has not been addressed as such in ref. 31. We can say however that a lower value of $\chi_{\text {polymer-fullerene }}$ is consistent with the fact that, so far, no tendency for amorphous demixing during casting (e.g. via spinodal decomposition) has been found for P3HT:fullerene blends, in contrast to some low band gap polymers:fullerene systems where this process was clearly observable. ${ }^{35}$

Since the often quoted value of the enthalpy of fusion of rr$\mathrm{P} 3 \mathrm{HT}\left(\Delta H_{\mathrm{c}}\right)$ of $16 \mathrm{~kJ} \mathrm{~mol}^{-1}$ reported by Malik et al. ${ }^{30}$ has recently been challenged to be about a factor two lower, ${ }^{33,36,37}$ it is of interest to compare fit results obtained while choosing various magnitudes for $\Delta H_{\mathrm{c}}$. The model seems to give a satisfactory fit for both an intermediate value of $\Delta H_{\mathrm{c}} \approx 12 \mathrm{~kJ} \mathrm{~mol}^{-1}$, and a high value in the vicinity of the one reported by Malik et al. In view of space and because of the fact that the order $\chi_{\text {P3HT-bisPCBM }}>$ $\chi_{\text {P3HT-РСBM }}>\chi_{\text {P3HT-ICBA }}$ is invariant with respect to the choice for $\Delta H_{\mathrm{c}}$, only the results obtained for $\Delta H_{\mathrm{c}} \approx 12 \mathrm{~kJ} \mathrm{~mol}^{-1}$ are given here. We do note, however, that $\Delta H_{\mathrm{c}} \approx 16 \mathrm{~kJ} \mathrm{~mol}^{-1}$ typically leads to a higher absolute value for $\chi_{\text {Р3нт-ICBA }}$ $\left(\chi_{\mathrm{P} 3 \mathrm{HT}-\mathrm{ICBA}}=0.3\right)$, while the other parameters remain more or less unchanged. As we will see, a lower value for $\Delta H_{\mathrm{c}}$ leads to a more pronounced thermodynamic contribution to the melting point depression, as it appears in the denominator in the RHS of eqn (2).

As a final remark, the apportioning coefficients $\psi_{0}$ and $\psi_{1}$ as well as the activation barrier for chain segmental motion at the crystalline-amorphous interface $\left(U_{\mathrm{P} 3 \mathrm{HT}}^{*}\right)$ are considered free floating. In case of $\Delta H_{\mathrm{c}} \approx 16 \mathrm{~kJ} \mathrm{~mol}^{-1}$, acceptable fits are achieved for $\psi_{0}=\psi_{1}=0.33$ for all fullerene derivatives. In case $\Delta H_{\mathrm{c}} \approx 12 \mathrm{~kJ} \mathrm{~mol}^{-1}$, the curvature in the graphs for bis-[60] PCBM and ICBA could only be reproduced satisfactory by assuming a significantly lower value for $\psi_{1}$.

In order to discriminate between kinetic vitrification and thermodynamic contributions, Fig. 1C also shows data corresponding to calculations assuming the following hypothetical cases: $(\mathrm{i}) \chi_{\text {fullerene-P3HT }}=\chi_{\mathrm{PCBM}-\mathrm{P} 3 \mathrm{HT}}=0.4$ (orange and green dashed thin lines), and (ii) $\chi_{\text {fullerene-P3HT }}=\chi_{\text {PCBM-P3HT }}=0.4$ and $T_{\mathrm{g}}($ fullerene $)=T_{\mathrm{g}}(\mathrm{rr}-\mathrm{P} 3 \mathrm{HT})$ (dotted lines) to complement the calculations providing the best fit result. Comparing the three data sets, we conclude that in all cases both the effect of thermodynamic and kinetic vitrification due to the presence of the fullerene is considerable. This exercise also shows that the thermodynamic contribution seems to be largely associated with the curvature in the melting point depression, whereas the effect of kinetic vitrification causes a more linear dependence on fullerene fraction. 
Interestingly, the results for ICBA suggest that in this case the thermodynamic contribution to melting point depression seems to dominate over the effect of vitrification associated with the high glass transition temperature. We note that the effect is reversed in case a higher value for $\Delta H_{\mathrm{c}}$ is used in the curve fitting, i.e. with concomitant decrease in interaction parameter differences. In other words, considering the approximate nature of the model, it is hard to determine which effect dominates under the actual experimental conditions or whether their contributions are comparable. Nevertheless, irrespective of the exact input parameter values the overall effect is that the growth of rr-P3HT crystals is hindered increasingly more effective in the order bis[60]PCBM < [60]PCBM < [60]ICBA.

The picture of [60]ICBA being the strongest vitrifier for rr-P3HT is supported experimentally when deducing the enthalpy of fusion $\left(\Delta H_{\mathrm{f}}^{\mathrm{P} 3 \mathrm{HT}}\right)$ of the rr-P3HT fraction in the various blends from the endothermic signals (Fig. 1D), which is directly correlated to the polymer's degree of molecular order and, hence, gives an indication of how strongly a given fullerene hinders the polymer's crystallisation: a lower $\Delta H_{\mathrm{f}}^{\mathrm{P} 3 \mathrm{HT}}$ signifies a stronger vitrifying effect and/or higher miscibility. For all three systems, there is a clear reduction in $\Delta H_{\mathrm{f}}^{\mathrm{P} 3 \mathrm{HT}}$ with increasing fullerene loading. The lowest $\Delta H_{\mathrm{f}}^{\mathrm{P} 3 \mathrm{HT}}$ is consistently observed for [60]ICBA blends, followed by binaries comprising [60]PCBM, with bis[60]PCBM containing systems always exhibiting the highest $\Delta H_{\mathrm{f}}^{\mathrm{P} 3 \mathrm{HT}}$. More specifically, for $60: 40$ (by weight) rr-P3HT : fullerene binaries, we measure the following enthalpies of fusion of $\mathrm{rr}-\mathrm{P} 3 \mathrm{HT}$ in the respective blends (compared to the neat system) indicative of different relative degrees of crystallinity: $\left[\Delta H_{\mathrm{f}}^{\mathrm{P} 3 \mathrm{HT}}\right.$ (blend) $/ \Delta H_{\mathrm{f}}^{\mathrm{P} 3 \mathrm{HT}}$ (neat polymer)] $=0.55,0.76,0.84$ for the systems comprising [60]ICBA, [60] PCBM and bis[60]PCBM. Increasing the fullerene content to 70 wt $\%$ decreases $\left[\Delta H_{\mathrm{f}}^{\mathrm{P} 3 \mathrm{HT}}\right.$ (blend) $/ \Delta H_{\mathrm{f}}^{\mathrm{P} 3 \mathrm{HT}}$ (neat polymer) $]$ even further to $0.23,0.68,0.74$ for rr-P3HT:[60]ICBA, rr-P3HT:[60] PCBM and rr-P3HT:bis[60]PCBM, respectively. All blends retain, though, a certain crystalline fraction of the polymer even at high content of the fullerenes.

We note in this context that the rr-P3HT:[60]ICBA solid solutions that form upon blending seemed to be strikingly stable. For instance, annealing rr-P3HT:[60]ICBA blends (30 : 70 weight ratio) at $165{ }^{\circ} \mathrm{C}$ for 1 hour did not lead to any increase in $\Delta H_{\mathrm{f}}^{\mathrm{P} 3 \mathrm{HT}}$. In strong contrast, for rr-P3HT:[60]PCBM and rr-P3HT:bis[60]PCBM binaries, an increase in the $\Delta H_{\mathrm{f}}^{\mathrm{P} 3 \mathrm{HT}}$ is recorded during an identical heat treatment, with the rr-P3HT:[60]PCBM blends exhibiting micron-sized fullerene crystals. This observation, in conjunction with the higher values found for $\Delta H_{\mathrm{f}}^{\mathrm{P} 3 \mathrm{HT}}$ suggests that the quenched amorphous solution is less stable in blends with [60]PCBM and bis[60] PCBM (respectively, squares and triangles) due to the stronger repulsive interaction between donor and acceptor, as represented by the higher Flory interaction parameters. While we ignore the fact that ICBA is a mixture of isomers, which may influence vitrification and the formation of an intermixed phase, we like to highlight that for bis-[60]PCBM, which is also such a mixture, we do not observe a strong vitrification/ intermixing.
Strikingly, the effect the different fullerenes have on the crystallization of rr-P3HT is reflected by the extent they quench the rr-P3HT's photoluminescence (PL), with the photoluminescence quenching (PLQ) giving a direct indication of the degree of molecular mixing of the two components (Fig. 2). Indeed, while all blends exhibit increased quenching with fullerene content, those comprising bis[60]PCBM consistently display the highest PL emission (i.e. the lowest PLQ) across the entire composition range, while the lowest PL (highest PLQ) is observed for binaries comprising [60]ICBA, with an interim behaviour found for [60]PCBM-containing blends. This suggests that the presence of more intimately mixed phases are present in rr-P3HT:[60]ICBA- and rr-P3HT:[60]PCBM-blends compared to rr-P3HT:bis[60]PCBM binaries.

Based on the PL data as well as our calculations, we suggest a structural picture where the polymer can crystallise to the greatest extent in the binary comprising bis[60]PCBM which has the lowest thermodynamic miscibility with rr-P3HT. This results in more prominent phase separation of this fullerene with rr-P3HT and, hence, the lowest amount of PL quenching. At the other side of the spectrum are the [60]ICBA-containing

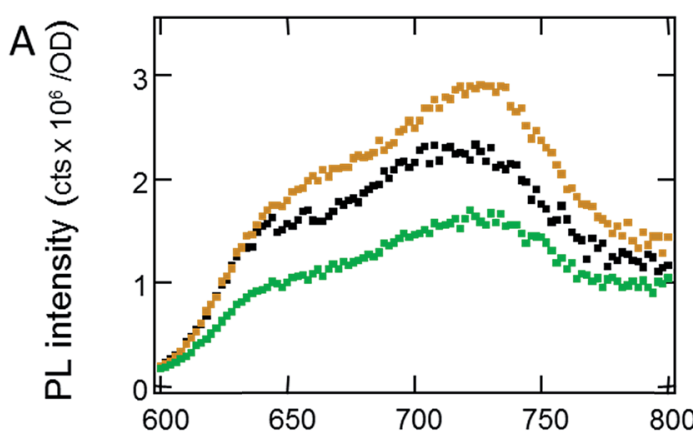

Wavelength $(\mathrm{nm})$

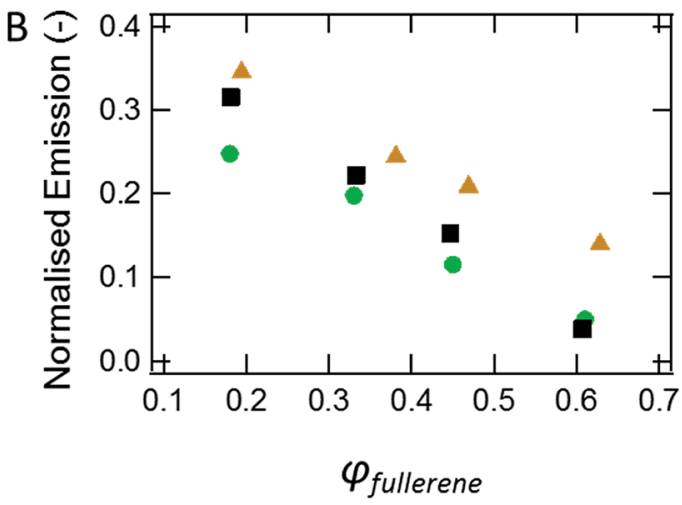

Fig. $2 \mathrm{PL}$ emission characteristics of pristine solution-cast rr-P3HT:fullerene blends, showing clear differences in emission intensity. (A) Corrected emission of blends comprising 55 wt\% rr-P3HT and [60]ICBA (green circles; equivalent to 34 vol\%), bis[60] PCBM (orange triangles; 38 vol\%) and [60]PCBM (black squares; 34 vol\%) respectively (corresponding to the optimised OPV devices discussed subsequently). (B) Normalised PL intensity of the different blends plotted as a function of composition. All data has been corrected for differences in number of absorbed photons at the excitation wavelength of $520 \mathrm{~nm}$ and normalised to the emission of neat rr-P3HT. 


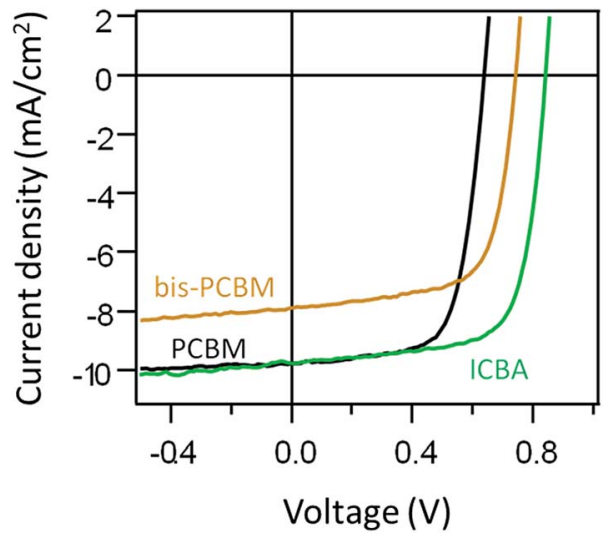

Fig. $3 \quad J-V$ characteristics of rr-P3HT:fullerene devices optimised for thickness and composition (rr-P3HT content: 55 wt\%) after annealing at $165^{\circ} \mathrm{C}$ for 7 minutes. [60]ICBA (green circles; equivalent to 34 vol\%), bis[60]PCBM (orange triangles; 38 vol\%) and [60]PCBM (black squares; 34 vol\%).

blends; of all studied fullerenes, [60]ICBA has the highest thermodynamic compatibility with rr-P3HT and also acts as the strongest vitrifier, inhibiting the crystallisation of rr-P3HT during the casting process most, in agreement with our PLQ data.

Intriguingly, the different tendencies towards kinetic and thermodynamic vitrification of the various blends seem to correlate with the $J_{\mathrm{sc}}$ of OPV devices fabricated with them ( $\sim 55$ wt\% rr-P3HT; Fig. 3). The highest $J_{\mathrm{sc}}$ is observed for the blends comprising the greatest fraction of solid solution (rr-P3HT:[60]ICBA and rr-P3HT:[60]PCBM), compared to the more prominently phase-separated rr-P3HT:bis[60]PCBM binary. Since the three systems had similar absorption profiles (Fig. 4, left) we can eliminate differences in number of absorbed photons as the origin of this difference in $J_{\mathrm{sc}}$. The Hecht expression $^{38}$ was therefore used to assess whether the differences in the microstructure affected recombination processes in the corresponding active layers (Fig. 4, right). Across the light intensities probed, all three systems exhibited generally mono- molecular recombination characteristics, implying a similar recombination mechanism in all cases. This is significant as it indicates that even with drastically varied fractions of finely intermixed and crystalline material, corroborated by grazingangle incidence wide-angle X-ray scattering (GIWAXS; see Fig. 5), recombination losses are comparable for all the rrP3HT:fullerene systems investigated. Based on our observations, we thus attribute the difference in $J_{\mathrm{sc}}$ of the devices to a change in conversion of the absorbed photons into free charge carriers. For the systems where smaller fractions of intermixed solid solution co-exist with phase-pure polymer (deduced from $\Delta H_{\mathrm{f}}^{\mathrm{P} 3 \mathrm{HT}}$ ) and likely phase-pure fullerene domains, less charges are generated, supporting the picture that exciton dissociation occurs in finely intermixed phases as long as minority fractions of a phase-pure donor and/or acceptor are present. ${ }^{39,40}$ In agreement with this model is also the fact that we observe changes in the fill factor indicating a slightly more bias-independent charge collection efficiency in the systems containing higher intermixed fractions; though it should be noted that the differences we observe are modest to draw full conclusions (from 0.66, 0.67 and 0.70 when changing the fullerene from bis [60]PCBM, [60]PCBM and [60]ICBA, respectively).

In summary, we have demonstrated that the crystallization behaviour of rr-P3HT as a polymeric component in solution-cast polymer:fullerene blends for organic PV strongly depends on the nature of the fullerene derivative. This is due to a [small] difference in their miscibility, but mainly because of a difference in how strongly the polymer gets vitrified, both due to thermodynamic and kinetic reasons, upon addition of the fullerene. Thermal analysis shows that the degree of crystallinity and the non-equilibrium melting point in solution-cast blend films is suppressed with increasing fullerene content, the effect becoming increasingly pronounced in the order of: bis[60]PCBM < [60]PCBM < [60]ICBA. Curve fitting the thermal analysis data using a combination of Lauritzen-Hoffman and Flory-Huggins theory reveals that the differences in melting point depression cannot be purely explained by thermodynamics, despite the fact that the miscibility of the fullerene derivatives with rr-P3HT increases in the same order. Instead,
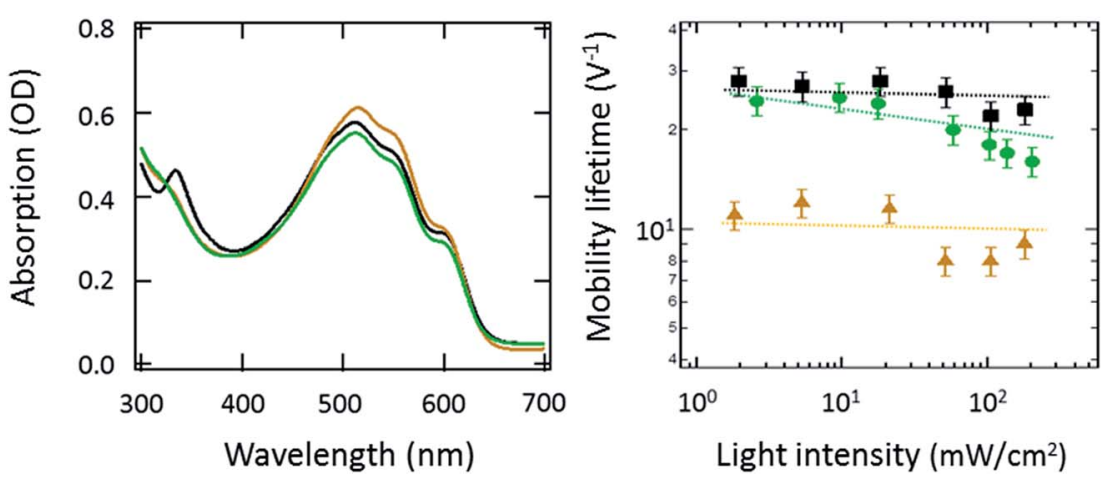

Fig. 4 Optoelectronic features of rr-P3HT:fullerene blends (rr-P3HT content: 55 wt\%). Left: Absorption spectra of rr-P3HT:fullerene blends prepared under the same conditions as those used for OPV devices, showing similar absorption profiles for all three systems. Right: Hecht analysis for devices made with such rr-P3HT:fullerene blends showing the mobility-lifetime product as a function of light intensity for systems comprising [60]PCBM (black squares; 34 vol\%), [60]ICBA (green circles; 34 vol\%) and bis[60]PCBM (orange triangles; 38 vol\%). All devices appear to exhibit relatively independent mobility lifetime as a function of light intensity. 
kinetic vitrification of the blends caused by the relatively high glass transition temperature of the fullerenes provides for an essential contribution to explain the overall effect. This phenomenon is likely to be even more pronounced in OPV blends comprising some of the new, high-performance donor polymers, such as PTB7 that already in the neat form often have a low tendency to crystallise. It also occurs in blends of PCE11 (i.e., PffBT4T-2OD), which has a strong tendency to aggregate in solution, ${ }^{\mathbf{4 1}}$ and [60]PCBM that have been demonstrate to display efficiencies $>10 \% .{ }^{41}$ Indeed, we find a pronounced cold-crystallisation exotherm in the first heating thermogram of a $1: 1$ PCE11 : [60]PCBM blend drop-cast at $100{ }^{\circ} \mathrm{C}$ (Fig. 6) - that is in a scenario where the solvent is extracted relatively fast - while for the two neat components (PCE11, [60]PCBM), no such feature was observed. Very similar to P3HT, the addition of the fullerene clearly hinders crystallisation of the PCE leading to at

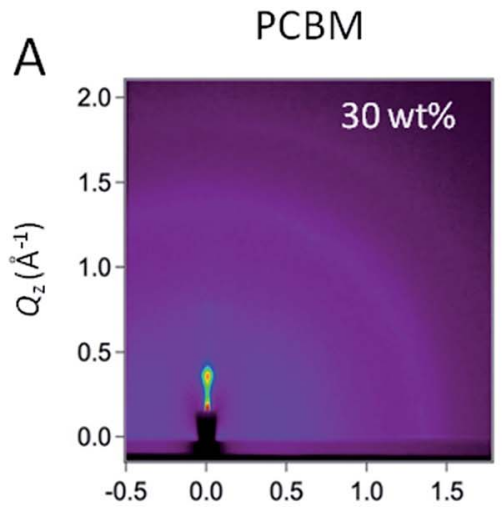

Bis-PCBM
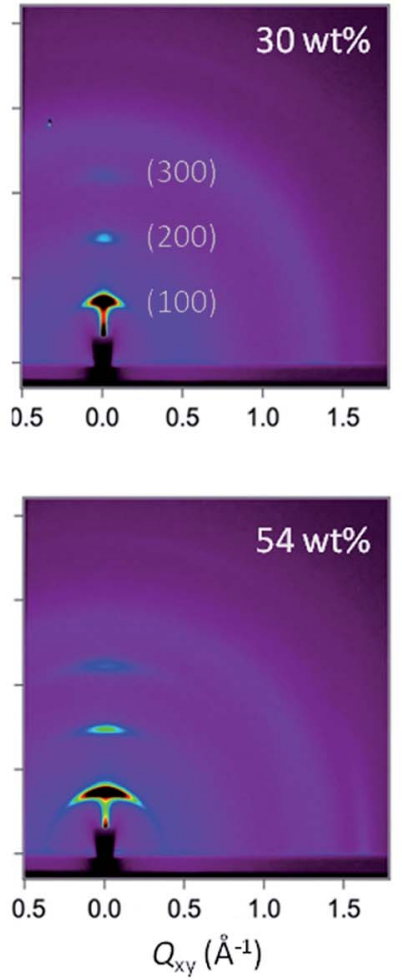

ICBA
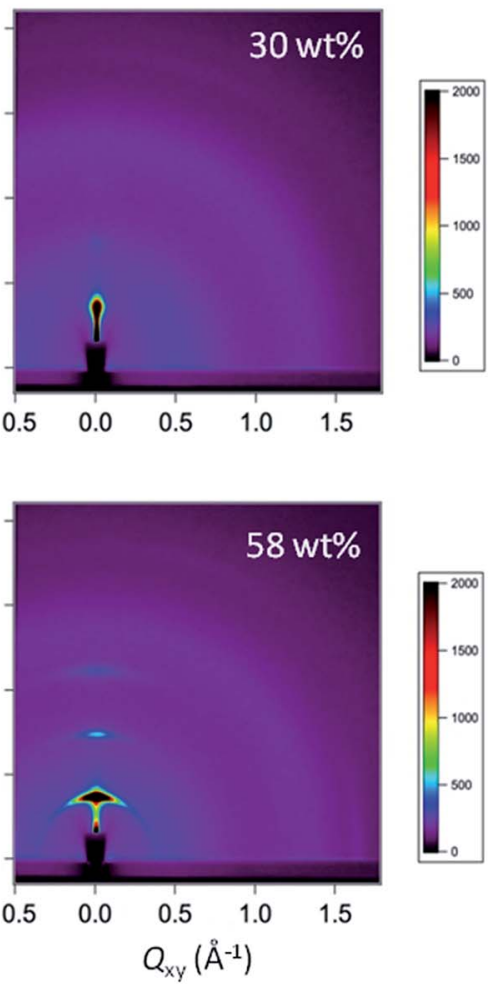
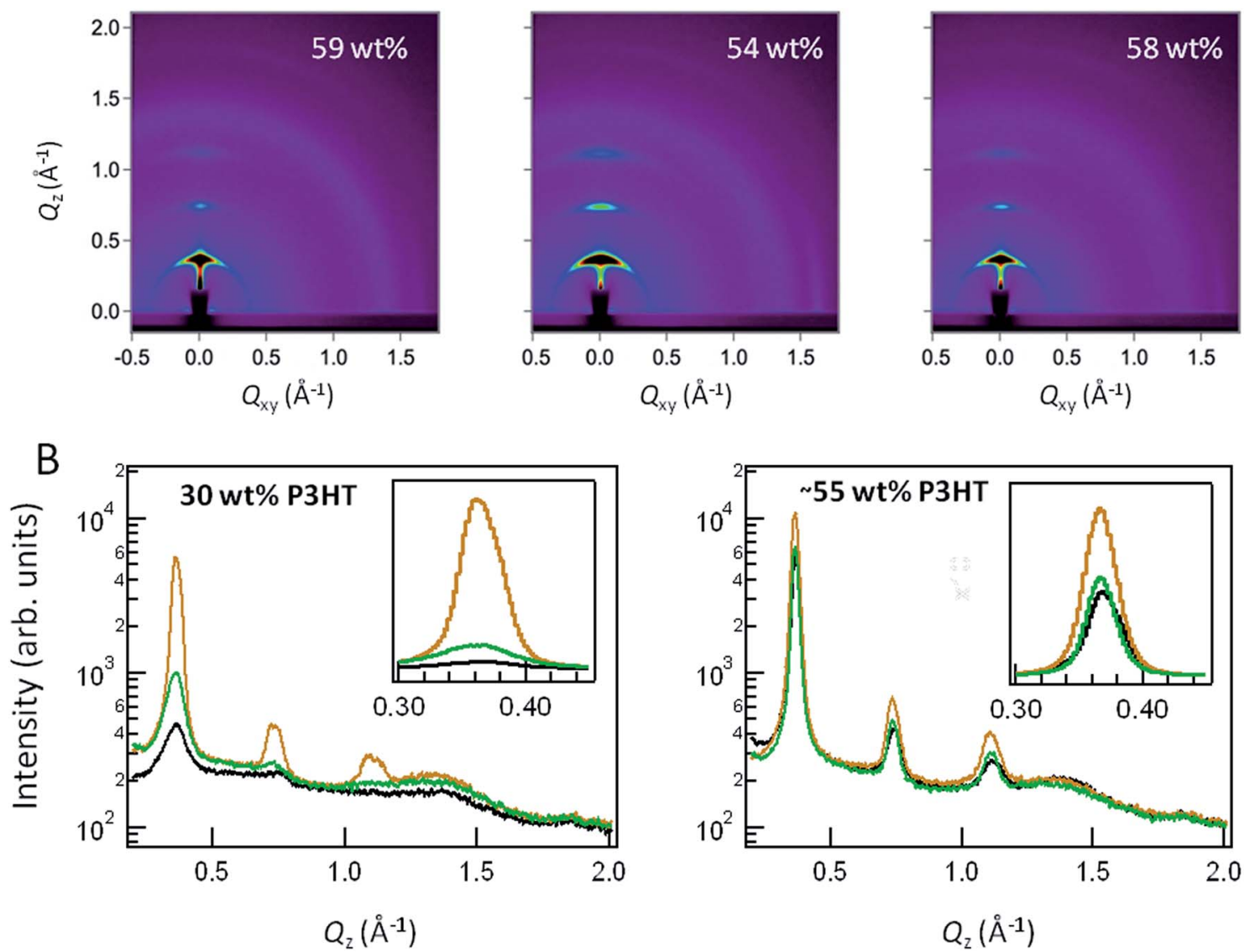

Fig. 5 GIWAXS data obtained for rr-P3HT:fullerene blends annealed at $165^{\circ} \mathrm{C}$, for 7 minutes. (A) 2D-scattering patterns of blends comprising 30 wt\% rr-P3HT (top; equivalent to 22, 24, and 22 vol\% [60]PCBM, bis[60]PCBM and [60]ICBA); and 55 wt\% rr-P3HT (bottom; equivalent to 34,38 and 34 vol\% of [60]PCBM, bis[60]PCBM and [60]ICBA). For reference, the positions of the lamellar reflections have been labelled in the middle graph. (B) Corresponding $Q_{z}$-integration of systems of a 30 (left) and $\sim 55$ (right) wt\% of rr-P3HT; the blends comprising [60]PCBM (black), bis[60] PCBM (orange) and [60]ICBA (green) show clear differences in scattering intensity from the lamellar reflection of $r$ - P3HT. Inset shows the (100) diffraction on a linear scale to aid comparison. 


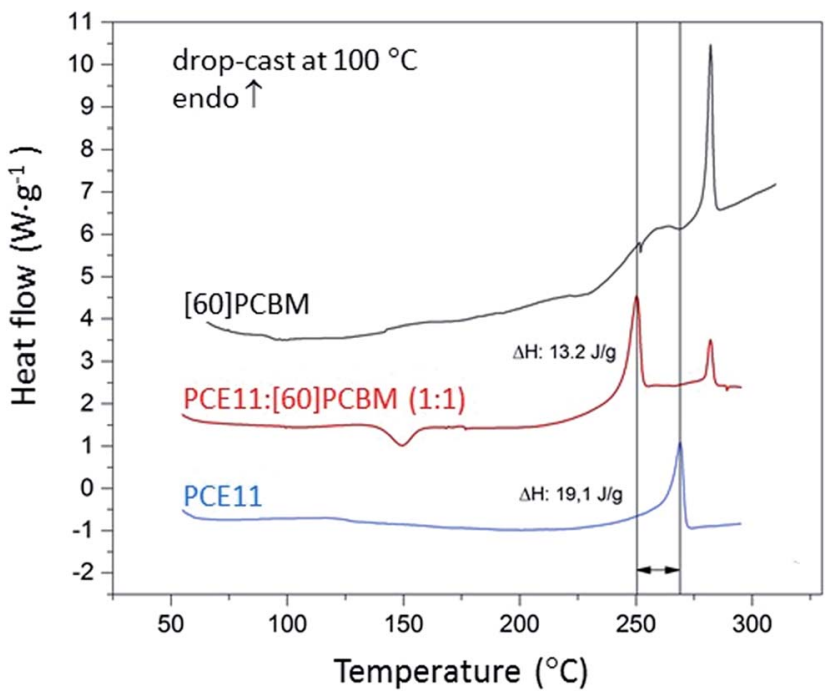

Fig. 6 Thermal behaviour of PCE11 (i.e. PffBT4T-2OD), [60]PCBM and a 1:1 blend of them. All systems were drop-cast at $100{ }^{\circ} \mathrm{C}$. A noticeable cold crystallisation exotherm is observed for the binary, while such an exothermic feature is missing in the neat components, highlighting that even for materials that can strongly aggregate in solution, such as PCE11, vitrification occurs upon blending with a fullerene.

least partial vitrification of the blend. The vitrification effect is pronounced; when casting the same system (PCE11:[60]PCBM) at room temperature and more time is given to phase separate, cold crystallisation still occurs in the blends (Fig. S1†), highlighting that even in systems where the donor can pre-aggregate in solution vitrification effects cannot be neglected.

Knowledge of vitrification, and the fact that not only thermodynamic arguments need to be considered, is important as presence (or absence) of a highly intermixed, (vitrified) phase significantly influences the optoelectronic processes within the resulting donor:acceptor blends, evidenced by the increase in exciton dissociation rate with the fraction of the intermixed (non-crystalline) phase. At the same time it is important to realize that efficient charge extraction relies on the presence of phase pure/crystalline regions. In case that crystallization of rr-P3HT is considerable, exciton dissociation becomes less efficient, as evidenced by a decrease in photoluminescence quantum yield and lower short circuit current. Overall, our results emphasize the delicate balance of phase compositions required for optimal device performance and highlight the importance of processing memory on the final microstructure, for which consideration of vitrification processes is of paramount importance. Indeed, both thermodynamic and kinetic factors influence vitrification of donor:acceptor blends - both factors that can be influenced by materials design and judicious choice of deposition conditions.

\section{Materials and methods}

\section{Materials}

We selected a rr-P3HT with a molecular weight close to a recently reported material (weight-average molecular weight,
$M_{\mathrm{w}}: 135 \mathrm{~kg} \mathrm{~mol}^{-1}, D: 1.9$, RR: $\left.99 \%\right)^{42}$ that had shown best performance amongst a range of P3HTs when blended with fullerenes and that can be expected to lead to a semicrystalline morphology with crystalline moieties embedded in an amorphous 'matrix'. ${ }^{43}$ It was prepared using the same route as described in ref. 42 , i.e. by a Grignard metathesis polymerisation at $55{ }^{\circ} \mathrm{C}$ with a $0.15 \mathrm{~mol} \%$ catalyst loading of $\mathrm{Ni}(\mathrm{dppp}) \mathrm{Cl}_{2}$. The polymer was purified by Soxhlet extraction with methanol and then extracted with chloroform. Weight-average molecular weight and dispersity $(D)$ were determined by size-exclusion chromatography (SEC) using an Agilent 1200 series GPC-SEC running chlorobenzene at $80^{\circ} \mathrm{C}$. The instrument was fitted with two PL gel mixed-B columns in series, and calibrated against polystyrene standards. Regioregularity (RR) was determined by integration of the alpha-methylene region in the ${ }^{1} \mathrm{H}$ NMR spectrum. PCE11 (i.e. PffBT4T-2OD) was purchased from Ossila Limited, UK with an $M_{\mathrm{n}}>55 \mathrm{~kg} \mathrm{~mol}^{-1}$ and a dispersity, $D=2.14$ (according to the data sheet provided). The fullerenes were supplied by Solenne BV.

\section{Blend fabrication}

Blend samples were prepared by dissolving the appropriate polymer : fullerene ratio $\left(20 \mathrm{mg} \mathrm{ml} \mathrm{m}^{-1}\right.$ total mass) in chlorobenzene to which $1.5 \mathrm{vol} \%$ chloronaphthalene was added. Spincoating was carried out at $1200 \mathrm{rpm}$ for $45 \mathrm{~s}$, followed by 2000 rpm for $7 \mathrm{~s}$. Optical samples were prepared on glass; GIWAXS samples on silicon wafer, and OPV devices on ITO glass as discussed below. For thermal analysis, films were drop-cast rather than spin coated.

\section{Device fabrication}

Glass substrates coated with ITO were cleaned in acetone in an ultrasonic bath, followed by sequential washing in deionised water comprising 2 vol\% soap, deionised water and isopropyl alcohol for 20 minutes each, followed by drying with nitrogen. A $40 \mathrm{~nm}$ layer of poly(3,4-ethylenedioxythiophene):poly(styrenesulfonate) (PEDOT:PSS) (Clevios PVP Al 4083) was coated via spin-coating at $4000 \mathrm{rpm}, 40 \mathrm{~s}$ and then dried on a hotplate at $165^{\circ} \mathrm{C}$ for $10 \mathrm{~min}$. rr-P3HT:fullerene blend solutions were subsequently deposited as described above. Solution concentration was varied between $19-23 \mathrm{mg} \mathrm{ml}^{-1}$ to vary the thickness of the active layer and blend ratios between $(1: 1$ and $1: 0.7$, polymer : fullerene by weight). The electrodes were then evaporated under vacuum $\left(<10^{-6}\right.$ Torr). Samarium was typically evaporated at around $0.7 \AA \mathrm{s}^{-1}$, until a thickness of $15 \mathrm{~nm}$ was attained. Aluminium was typically evaporated at around $3 \mathrm{~A} \mathrm{~s}^{-1}$, until a layer around $75 \mathrm{~nm}$ thick was attained. Each substrate contained 5 solar cells each of which had an area of $0.06 \mathrm{~cm}^{2} . J-V$ characteristics of the devices were measured at 1 sun (AM 1.5G) under a nitrogen atmosphere. A xenon lamp (Newport) and Keithley 2408 SMU were utilised for the measurement. The light intensity dependant $J-V$ characteristics were measured using an array of 14 red $1 \mathrm{~W}$ LEDs under nitrogen. Light and dark measurements were measured sequentially using a Keithley 2400 source meter unit. As previously reported $V_{\text {oc }}$ of around 0.64, 0.74 and $0.84 \mathrm{~V}$ were observed for [60]PCBM, bis[60] 
PCBM and [60]ICBA respectively, due the successively higher LUMOs these materials.

\section{Differential scanning calorimetry}

Differential scanning calorimetry was carried out on films that had been drop-cast onto glass slides and then dried under vacuum for 4 days to remove residual solvent, following procedures described in ref. 44. The films were scratched from the substrates and placed into a DSC pans. Heating was carried out at $10{ }^{\circ} \mathrm{C} \min ^{-1}$ over a temperature regime of $40-270{ }^{\circ} \mathrm{C}$ under a nitrogen atmosphere, utilising a Mettler Toledo DSC822. Phase diagrams were constructed from the end-of-melting temperature as also described in ref. 44. We note, the P3HT:[60] PCBM binary displayed a very similar phase behaviour as described in ref. 44, with a eutectic composition around 35 to $40 \mathrm{wt} \%$. The eutectic temperature is not as sharp as in ref. 44, which we attribute to the higher molecular weight-nature of the donor polymer we used here. We also note that we could not measure the melting point depression of the fullerene in systems comprising ICBA or [60]bis-PCBM as these two fullerenes did not display a notable melting temperature even when used in their neat form.

\section{Steady-state photoluminescence (PL)}

All measurements utilised a Horiba Spex Fluormax-1 spectrofluorometer with an acquisition angle of $60^{\circ}$. The intensity of emission was corrected for the density of absorbed photons using the following equation:

$$
\mathrm{PL}_{\text {corr }}=\mathrm{PL}_{\text {raw }} /\left(1-10^{-A}\right)
$$

where $A$ is the absorption at the excitation wavelength. All samples were excited at $520 \mathrm{~nm}$ and emission measured between $540-800 \mathrm{~nm}$.

\section{Acknowledgements}

We are very grateful to the UK's Engineering and Physical Sciences Research Council (EPSRC) for financial support via the Doctoral Training Centre in Plastic Electronics EP/G037515/ 1(PW, NS, JdM and JB), the EPSRC platform grant EP/G060738/1 (NS). JB holds an Industrial Fellowship with the Royal Commission for the Exhibition of 1851. We furthermore acknowledge the ACS Petroleum Fund (New Directions Grant). NS is also supported by a European Research Council (ERC) Starting Independent Researcher Fellowship under the grant agreement no. 279587.

\section{References}

1 M. A. Faist, S. Shoaee, S. Tuladhar, G. F. A. Dibb, S. Foster, W. Gong, T. Kirchartz, D. D. C. Bradley, J. R. Durrant and J. Nelson, Adv. Energy Mater., 2013, 3, 744-752.

2 (a) P. A. Troshin, H. Hoppe, J. Renz, M. Egginger, J. Y. Mayorova, A. E. Goryochev, A. S. Peregudov, R. N. Lyubovskaya, G. Gobsch, N. S. Sariciftci and
V. F. Razumov, Adv. Funct. Mater., 2009, 19, 779-788; (b) C. M. Amb, S. Chen, K. R. Graham, J. Subbiah, C. E. Small, F. So and J. R. Reynolds, J. Am. Chem. Soc., 2011, 133, 10062-10065; (c) Z. Fei, R. S. Ashraf, Z. Huang, J. Smith, R. J. Kline, P. D'Angelo, T. D. Anthopoulos, J. R. Durrant, I. McCulloch and M. Heeney, Chem. Commun., 2012, 48, 2955-2957.

3 A. A. Y. Guilbert, L. X. Reynolds, A. Bruno, A. MacLachlan, S. P. King, M. A. Faist, E. Pires, J. E. Macdonald, N. Stingelin, S. A. Haque and J. Nelson, ACS Nano, 2012, 6, 3868-3875.

4 (a) N. D. Treat, A. Varotto, C. J. Takacs, N. Batara, M. AlHashimi, M. J. Heeney, A. J. Heeger, F. Wudl, C. J. Hawker and M. L. Chabinyc, J. Am. Chem. Soc., 2012, 134, 1586915879; (b) B. A. Collins, Z. Li, C. R. McNeill and H. Ade, Macromolecules, 2011, 44, 9747-9751.

5 (a) M. Lenes, G.-J. A. H. Wetzelaer, F. B. Kooistra, S. C. Veenstra, J. C. Hummelen and P. W. M. Blom, Adv. Mater., 2008, 20, 2116-2119; (b) Y. He, H.-Y. Chen, J. Hou and Y. Li, J. Am. Chem. Soc., 2010, 132, 1377-1382.

6 D. Leman, M. A. Kelly, S. Ness, S. Engmann, A. Herzing, C. Snyder, H. W. Ro, R. J. Kline, D. M. DeLongchamp and L. J. Richter, Macromolecules, 2015, 48, 383-392.

$7 \mathrm{~A}$ value of $\rho_{\mathrm{P} 3 \mathrm{HT}}=1.0 \mathrm{~g} \mathrm{~cm}^{-3}$ likely represents the amorphous fraction somewhat better than the crystalline fraction. However, here we ignored the small increase in the average mass density of P3HT upon increasing the amount of crystalline material; see for instance: F. Machui, S. Rathgeber, N. Li, T. Ameri, C. J. Brabec, J. Mater. Chem., 2012, 22, 15570-15577 (ESI $\dagger$ ). A value of $\rho_{\mathrm{P} 3 \mathrm{HT}}=1.1 \mathrm{~g}$ $\mathrm{cm}^{-3}$, for instance, did not change the outcome of the calculations performed in the present work.

8 C. W. T. Bulle-Lieuwma, W. J. H. van Gennip and J. K. J. van Duren, Appl. Surf. Sci., 2003, 203-204, 547.

9 A slightly lower value for the mass density of PCBM of $\rho=1.3$ $\mathrm{g} \mathrm{cm}^{-3}$ has been published in: J. W. Kiel, B. J. Kirby, C. F. Majkrzak, B. B. Maranville and M. E. Mackay, Soft Matter, 2010, 6, 641-646.

10 P. J. Flory, Principles of Polymer Chemistry, Cornell University Press, 1953.

11 Y. Kim, S. A. Choulis, J. Nelson, D. D. C. Bradley, S. Cook and J. R. Durrant, J. Mater. Sci., 2005, 40, 1371-1376.

12 P. B. Rim and J. P. Runt, Macromolecules, 1984, 17, 15201526.

13 (a) R. L. Scott, J. Chem. Phys., 1949, 279; (b) T. Nishi and T. T. Wang, Macromolecules, 1975, 8, 909-915.

14 If one of the components represents a considerable amount of a solvent, $T_{\mathrm{m}}^{\mathrm{o} \prime}$ can be interpreted as a dissolution temperature.

15 M. J. M. Wirix, P. H. H. Bomans, H. Friedrich, N. A. J. M. Sommerdijk and G. de With, Nano Lett., 2014, 14, 2033-2038.

16 (a) J. I. Lauritzen Jr and J. D. Hoffman, J. Appl. Phys., 1973, 44, 4340-4352; (b) J. Hoffman, G. T. Davis and J. Lauritzen Jr, in Treatise on Solid State Chemistry, ed. N. B. Hannay, Springer US, 1976, pp. 497-614; (c) J. P. K. Doye and D. Frenkel, Phys. Rev. Lett., 1998, 81, 2160-2163. 
17 (a) Q. Guo and G. Groeninckx, Polymer, 2001, 42, 8647-8655;

(b) G. Siqueira, C. Fraschini, J. Bras, A. Dufresne, R. Prud'homme and M.-P. Laborie, Eur. Polym. J., 2011, 47, 2216-2227.

18 (a) M. M. Abolhasani, M. Naebe and Q. Guo, Phys. Chem. Chem. Phys., 2014, 16, 10679-10687; (b) M. Abolhasani, M. Rezaei Abadchi, K. Magniez and Q. Guo, J. Therm. Anal. Calorim., 2015, 119, 527-536.

19 G. W. Heffner and D. S. Pearson, Macromolecules, 1991, 24, 6295-6299.

20 J. Liu, M. Arif, J. Zou, S. I. Khondaker and L. Zhai, Macromolecules, 2009, 42, 9390-9393.

21 (a) R. D. K. Misra, D. Depan, V. S. A. Challa and J. S. Shah, Phys. Chem. Chem. Phys., 2014, 16, 19122-19129; (b) Y. Luo, F. A. Santos, T. W. Wagner, E. Tsoi and S. Zhang, J. Phys. Chem. B, 2014, 118, 6038-6046.

22 (a) J. D. Hoffman, Polymer, 1982, 23, 656-670; (b) An expression for $N_{0}$ has been given by Hoffman (J. D. Hoffman, Polymer, 1982, 23, 656): $N_{0}=k_{\mathrm{s}} z C_{\mathrm{p}} P_{0}$. Here $k_{\mathrm{s}}$ is the number of stems in the growth front, $z$ is the number of crystallizing repeat units in $\mathrm{L}, C_{\mathrm{p}}$ is a coordination number in the range 4-6 and $P_{0}$ is the configurational path degeneracy accounting for the occurrence of different paths for a stem to attach to the growth front. If no fluctuations in stem length are considered $P_{0}=1$.

$23 B=B_{1}$ only holds as long as the apportioning coefficient is the same for both detachment rates.

24 (a) J. D. Hoffman and R. L. Miller, Polymer, 1997, 38, 31513212; (b) This particular expression invokes a Vogel-type temperature dependence. In case $T \gg T_{\mathrm{g}}$ an Arrhenius factor is better suited.

25 J. D. Hoffman, C. M. Guttman and E. A. DiMarzio, Faraday Discuss. Chem. Soc., 1979, 68, 177-197.

26 Tacit assumption is that primary and secondary nucleation are treated as separate regimes.

27 This expression is consistent with regime I-type growth in LH terminology, in which the substrate completion rate is significantly higher than the secondary nucleation rate, resulting in highly ordered chain folded crystals, as expected under the present conditions. M. M. Abolhasani, M. R. Abadchi, K. Magniez and Q. Guo, J. Therm. Anal. Calorim., 2015, 119, 527.

28 M. Aubin and R. E. Prud'homme, Macromolecules, 1988, 21, 2945-2949.

29 As the model does not take recrystallization into account we are somewhat hesitant to consider the calculated end-of- melting points in our comparison with the experimental melting temperatures. Instead we have normalized all melting temperatures to that of neat, pristine P3HT.

30 S. Malik and A. K. Nandi, J. Polym. Sci., Part B: Polym. Phys., 2002, 40, 2073-2085.

31 D. R. Kozub, K. Vakhshouri, L. M. Orme, C. Wang, A. Hexemer and E. D. Gomez, Macromolecules, 2011, 44, 5722-5726.

32 T. T. Ngo, D. N. Nguyen and V. T. Nguyen, Adv. Nat. Sci.: Nanosci. Nanotechnol., 2012, 3, 045001.

33 F. P. V. Koch, M. Heeney and P. Smith, J. Am. Chem. Soc., 2013, 135, 13699-13709.

34 J. D. Hoffman and J. J. Weeks, J. Res. Natl. Bur. Stand., Sect. A, 1962, 66, 13.

35 S. Kouijzer, J. J. Michels, M. van den Berg, V. S. Gevaerts, M. Turbiez, M. M. Wienk and R. A. J. Janssen, J. Am. Chem. Soc., 2013, 135, 12057 and references cited therein.

36 C. R. Snyder, R. C. Nieuwendaal, D. M. DeLongchamps, C. K. Luscombe, P. Sista and S. D. Boyd, Macromolecules, 2014, 47, 3942-3950.

37 C. S. Lee and M. D. Dadmun, Polymer, 2014, 55, 4-7.

38 R. A. Street and M. Schoendorf, Phys. Rev. B: Condens. Matter Mater. Phys., 2010, 81, 205307.

39 (a) F. C. Jamieson, E. B. Domingo, T. McCarthy-Ward, M. Heeney, N. Stingelin and J. R. Durrant, Chem. Sci., 2012, 3, 485-492; (b) E. Buchaca-Domingo, A. J. Ferguson, F. C. Jamieson, T. McCarthy-Ward, S. Shoaee, J. R. Tumbleston, O. G. Reid, L. Yu, M. B. Madec, M. Pfannmoller, F. Hermerschmidt, R. R. Schroder, S. E. Watkins, N. Kopidakis, G. Portale, A. Amassian, M. Heeney, H. Ade, G. Rumbles, J. R. Durrant and N. Stingelin, Mater. Horiz., 2014, 1, 270-279.

40 P. Westacott, J. R. Tumbleston, S. Shoaee, S. Fearn, J. H. Bannock, J. B. Gilchrist, S. Heutz, J. deMello, M. Heeney, H. Ade, J. Durrant, D. S. McPhail and N. Stingelin, Energy Environ. Sci., 2013, 6, 2756-2764.

41 Y. Liu, J. Zhao, Z. Li, C. Mu, W. Ma, H. Hu, K. Jiang, H. Lin, H. Ade and H. Yan, Nat. Commun., 2014, 5, 5293.

42 J. H. Bannock, N. D. Treat, M. Chabinyc, N. Stingelin, M. Heeney and J. de Mello, Sci. Rep., 2016, 6, 23651.

43 M. Brinkmann and P. Ramnou, Adv. Funct. Mater., 2007, 17, 101.

44 C. Müller, T. A. M. Ferenczi, M. Campoy-Quiles, J. M. Frost, D. D. C. Bradley, P. Smith, N. Stingelin-Stutzmann and J. Nelson, Adv. Mater., 2008, 20, 3510. 